\title{
Real-Space Simulation of Electron Scattering in Imperfect Crystals and Reconstruction of the Electrostatic Potential.
}

\author{
Wouter Van den Broek ${ }^{1}$ and Christoph T. Koch ${ }^{1}$. \\ ${ }^{1 .}$ Institute for Experimental Physics, Ulm University, Ulm, Germany.
}

The Bloch-wave approach [1] is a powerful and efficient tool for the simulation of diffraction patterns of ideal crystals. However, when deviations from perfect crystallinity are present, like strain fields, defects or interfaces for instance, only approximate solutions are possible anymore. The multislice algorithm [1] (MSA) is preferable in these cases because it does not require any additional approximations for imperfect structures since it is based in real space.

In order to achieve diffraction data up to high angles while at the same time resolving fine details like HOLZ lines, a small sampling distance and a large extent must be combined in real space. Furthermore, to accommodate to arbitrary specimen tilts or to the non-periodicity brought about by strain fields, defects or interfaces, small slicing intervals of the order of 0.01 to $0.1 \mathrm{~nm}$ are necessary. As a result, the demands on computer memory and processing speed are high.

The improvement of graphics processing units (GPUs) in the last years has allowed to combine these two demands. Here, our forward dynamical electron scattering (FDES) software [2] is used. FDES is written in the CUDA programming language, with the CUFFT, CUBLAS and CURAND libraries and can simulate any combination of specimen-tilt series, beam-tilt series or focal series of high resolution transmission electron microscopy (HRTEM) images, diffraction patterns or convergent beam electron diffraction (CBED) patterns. Thermal diffuse scattering is accounted for with the frozen phonon approach.

In Figure 1 FDES's capabilities are demonstrated by computing CBED patterns of a PbSe-CdSe coreshell particle with 1963 atoms. The cubic particle's structure is based on CdSe rock-salt. The unit-cell parameter, $a$, measures $0.61 \mathrm{~nm}$. Pb-atoms are replacing the Cd-atoms in the cube's interior octahedron and are displaced by $[-a / 4, a / 4, a / 4]$. The acceleration voltage is $40 \mathrm{kV}$ and the convergence semi-angle is 15 mrad. The specimen is 1000 by 1000 pixels of $0.01 \mathrm{~nm}$ wide, resulting in a $0.1 \mathrm{~nm}^{-1}$ sampling in reciprocal space. The slice thickness is $0.01 \mathrm{~nm}$. An average is taken over 50 frozen phonon configurations; the computation time is $247 \mathrm{~s}$ on a NVIDIA Tesla K20 GPU, i.e. less than $5 \mathrm{~s}$ per configuration. The Debye-Waller factors for Se, $\mathrm{Cd}$ and $\mathrm{Pb}$ equal 0.0118, 0.0157 and $0.0217 \mathrm{~nm}^{2}$ respectively. Preliminary results have shown that samples of over a million atoms can be simulated as well.

Furthermore, FDES is an ideal test ground for inverse dynamical electron scattering (IDES), introduced by the authors in $[3,4]$, where the object's three-dimensional electrostatic potential distribution can be retrieved from a tilt series of TEM recordings, for example from HRTEM, CBED or scanning confocal electron microscopy. Since IDES is based on the reformulation of MSA as an artificial neural network, it takes the dynamical scattering of the electrons into account exactly.

A HRTEM tilt series is simulated of the PbSe-CdSe core-shell particle described above. The alpha tilt is varied from $-10^{\circ}$ to $+10^{\circ}$ in $2^{\circ}$ increments while the beta tilt is held at $0^{\circ}$. Then the beta tilt is varied 
from $-10^{\circ}$ to $+10^{\circ}$ in $2^{\circ}$ increments while the alpha tilt is held at $0^{\circ}$. The acceleration voltage is $80 \mathrm{kV}$, the focus and spherical aberration are $-20 \mathrm{~nm}$ and $64.2 \mu \mathrm{m}$, respectively, resulting in a point resolution of $0.17 \mathrm{~nm}$. The specimen is 600 by 600 pixels of $0.025 \mathrm{~nm}$ wide and 250 slices of $0.02 \mathrm{~nm}$ tall. The dose is 100 electrons per pixel. Partial spatial coherence and the CCD's modulation transfer function are included as well.

In order to save computation time, the IDES reconstruction is carried out with coarser parameters than the forward simulation: a slice thickness of $0.1 \mathrm{~nm}$ and an approximation to the specimen tilt by a shifted Fresnel propagator instead of the exact crystal tilts in FDES. Despite these coarser settings, an atomic-resolution reconstruction was obtained. Furthermore, $\ell_{1}$-norm regularization is applied to the object and sparseness is increased with a generalized potential. Mass contrast was observed in the reconstruction so that by applying a threshold to the reconstructed intensities, the $\mathrm{Pb}$-core could be isolated; see Figure 2.

MSA is preferable for the simulation of imperfect crystalline structures. To meet the computational demands which come with these real-space calculations we presented GPU-based MSA software that is capable of accurate and fast computation of finely sampled large specimens. Its capabilities were demonstrated with the simulation of a CBED pattern of a core-shell particle and the simulation of a HRTEM tilt-series that was subsequently used in the three-dimensional reconstruction routine IDES. [5]

\section{References:}

[1] EJ Kirkland in “Advanced Computing in Electron Microscopy”, (Springer, New York) p. 115.

[2] W Van den Broek and CT Koch, IMC Proceedings (2014), IT-16-P-2748.

[3] W Van den Broek and CT Koch, Phys. Rev. Lett. 109 (2012), p. 245502.

[4] W Van den Broek and CT Koch, Phys. Rev. B 87 (2013), p. 184108.

[5] The authors acknowledge the Carl Zeiss Foundation and the German Research Foundation (DFG, Grant No. KO 2911/7-1).
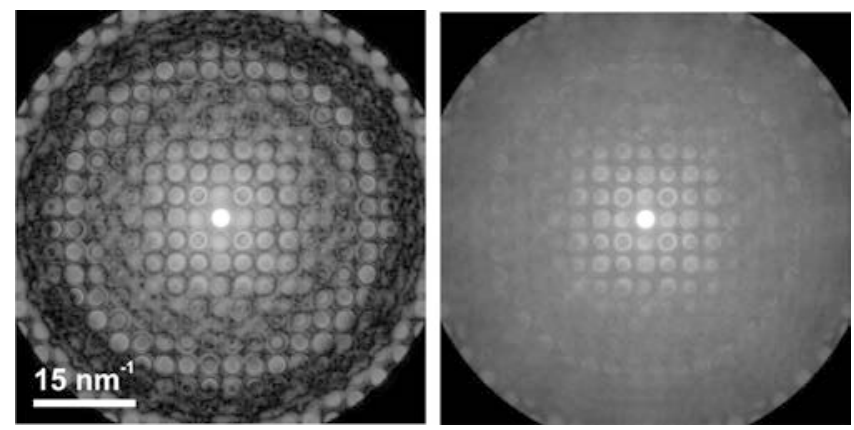

Figure 1. Simulation of a (001) PbSe-CdSe coreshell particle without (left) and with (right) thermal diffuse scattering included. Logarithmic gray scale.

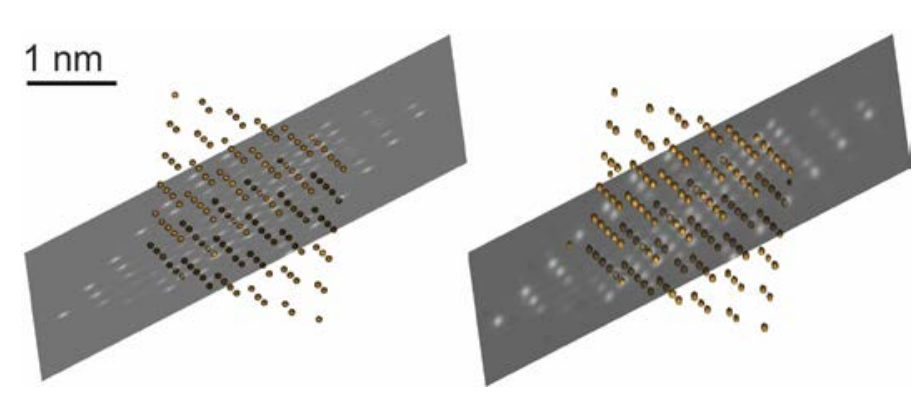

Figure 2. Left, the Pb-core of the PbSe-CdSe coreshell particle. Right, the reconstructed core, retrieved by thresholding and thus demonstrating mass contrast. 